\title{
Solubility Property of Baganuur Coal: Performance Assessment by FTIR Spectroscopic Analysis
}

\author{
Munkhtsetseg Sambuu ${ }^{1, a *}$, Khandmaa Tsagaanaa ${ }^{1, b}$, \\ Erdene-Ochir Ganbold ${ }^{1, \mathrm{c}}$, Galbadrakh Ragchaa $^{1, \mathrm{~d}}$, Enkhtor Sukhbaatar ${ }^{1, \mathrm{e}}$, \\ A. N. Oleshkevich ${ }^{2, f}$, and N. M. Lapchuk ${ }^{2, g}$ \\ ${ }^{1}$ Department of Physics, School of Arts and Sciences, Natural Sciences Division, National \\ University of Mongolia, Ulaanbaatar, Mongolia \\ ${ }^{2}$ Department of Physics, Belarusian State University, Minsk, Belarus \\ amunkhtsetseg_s@num.edu.mn, bkhandmaa@num.edu.mn, cerdeneochir_g@num.edu.mn, \\ dgalbadrakh@num.edu.mn, eenkhtur_s@num.edu.mn, flapchuk@bsu.by, '.Lapchuk@bsu.by
}

Keywords: Coal, Pyridine, Ionic Liquid, FTIR

\begin{abstract}
In the present work, the extraction of Mongolian Baganuur coal in solvents as pyridine and ionic liquid with 1-butyl-3-methyl-imidazolium chloride $([\mathrm{Bmim}] \mathrm{Cl})$ anion was first applied. The as recieved coal, its extracts and insoluble residues were then characterized using the Fourier transform infrared (FTIR) spectroscopy. The obtained FTIR spectra have revealed many new features in the field of coal study. An appearance or sharpening of the particular bands after the chemical treatment allow a determination of inactive or weak fundamental vibrations precisely. Some emphasis are as follows, substantial quantitative change, the integrated area decrease of water molecule band at $3260 \mathrm{~cm}^{-1}$ comparing to as received sample and ionic liquid treated extract, can be seen for the extract spectrum in the pyridine treatment. Pyridine react to coal structure particularly in long-wave frequency zone means very susceptible to the oxygen containing functional group. Upon interaction between acidic group of the coal and the basic solvent as pyridine, the inter-fragment hydrogen and ester bonding in the coal structure is breaking, thus increasing the solubility of the individual fragments via producing new components.

Towards forming $\mathrm{H}$ bond in the short wave zone $\mathrm{Cl}^{-}$anion shows a strong effect on the coal molecular structure. A stabilization of hydrogen bonds show well fluidization and a strong intermolecular interaction of the process via its powerful spectral intensity that is followed many new bands and considerable strengthening of band spectral integral area in this frequency region. In long-wave vibrational region there are appearances of many new bands, shift in frequency and depletion of the as recieved coal bands. $[\mathrm{Bmim}] \mathrm{Cl}$ treatment exhibits the highest effect of the disruption on the carboxylic acids dimer.
\end{abstract}

\section{Introduction}

Brown coal reserves around the capital city Ulaanbaatar is a great extent for its increasing demand of energy usage. In one of the local coal basins Baganuur, a brown coal reserve is estimated over half million metric tonnes containing 14 coal layers of which only 3 are under mining [1,2,3]. Currently, the deposit supplies $40 \%$ of the domestic demand for steam coal [3,4] for the coal fired power stations and traditional ger districts due to its convenient location and lower mining cost. Since the usage is mainly coal direct burning, this justifies the candidacy of an air pollution as supplementary source in particularly long winter months (corresponding $P M_{10-2.5}$ are over 300 and $100 \mu \mathrm{gm}^{-3}$ [5]). In this point of view an obstacle to further development of coal usage e.g. an improvement of the quality of coal, is terribly required. Several studies $[6,7,8,9,10,11,12,13]$ and etc. were done previously in which include Baganuur coal pyrolysis $[10,11]$ and the coalification [12]. However it is still essential to find an optimal experimental condition to maximize the conversion processes of Mongolian coals. Coal is itself highly heterogeneous, macromolecular matrix and its concept of structures still not completely accurate. The actual study of extraction process and the extraction product itself gives detailed information about the understanding of coal matrix. 